\title{
APPLICATION RESEARCH OF LOD TECHNOLOGY AND THE SHORTEST PATH ALGORITHM IN TRAFIC GEOGRAPHIC INFORMATION SYSTEM
}

\author{
Zhang Xunhu ${ }^{1}$, Zhang Xunlan ${ }^{2}$, Zhang He ${ }^{1}$ \\ ${ }^{1}$ National Quality Inspection and Testing Center for Surveying and Mapping Products, Beijing 100830, China - 8893784@qq.com; \\ 3885987@qq.com \\ ${ }^{2}$ Renfeng Town Central Primary School, Jiyang District, Jinan City, Shandong Province 251414, China - 1074049272@qq.com
}

\section{Commission IV, WG IV/10}

KEY WORDS: GIS, Traffic , LOD, Shortest path, Three-dimensional simulation

\begin{abstract}
:
3D visualization theory and technology has become a new research topic in the field of GIS and digital mapping. Based on a brief analysis of the development status of 3D GIS at home and abroad, this paper introduces the key technologies such as LOD technology and shortest path algorithm commonly used in traffic 3D GIS. The three-dimensional visualization technology in the application of traffic in the field of three-dimensional road network generation, vehicle navigation, simulation driving, etc., the analysis and summary of the vehicle collision model based on driving vehicles to detect road conditions and issue vehicle driving operation instructions, to some extent The simulated driving of the vehicle is realized. Based on the actual 3D geographic information platform, the fusion processing and 3D display of different precision terrain data are realized, and the function modules such as vehicle navigation and simulation driving are developed. The initial practice of LOD technology and shortest path algorithm in traffic 3D information system application was carried out.
\end{abstract}

\section{INTRODUCTION}

Visualization Theory and Technology Used in Cartography and GIS Since the early 1990s, GIS researchers have begun to introduce the theory and technology of computer graphics into the study of spatial data visualization. The visualization technology of 2D GIS is now basically mature.

Visualization is an important research content of 3D GIS. It mainly focuses on the reconstruction of terrain surface and the establishment of geometric model of building architecture, especially in the expression of terrain. Early multi-resolutions were generated using triangulation based on TIN hierarchy.

In recent years, with the increasing demand for 3D GIS, some GIS software companies have invested in research and development. Such as ESRI's ArcView 3D Analyst extension, Intergarph's GeoMedia Terrain module, and Gio's CCGIS. Their functions are mainly focused on the expression of terrain and architecture, attribute query, visual observation, visual analysis, spatial measurement and so on. It has a good application effect in a small area and appropriate data volume, but it is still insufficient for real-time and real-time visualization of largescale, complex environments and massive data. Some scholars have begun to study large-scale, multi-dimensional virtual reality research of three-dimensional geographic information, including data storage and data display. LOD technology is one of the most critical technologies in many studies. The shortest path algorithm is a key technology for 3D geographic information network analysis. It has a wide range of applications in path analysis, location analysis, and cost analysis. The combination of LOD technology and shortest path algorithm in traffic information system is one of the relatively new research fields.
The content of this paper is based on the three-dimensional geographic information platform of Beijing Intergraph Information Technology Co., Ltd., using LOD technology and shortest path algorithm to realize three-dimensional navigation, visual display and vehicle simulation driving in traffic geographic information system. The platform has the ability of large data volume spatial information processing, database driving, simulation implementation, cross-platform communication, secondary development support, etc., and can provide better 3D geographic information and virtual reality solutions. At present, it has been widely used in digital cities, digital communities, small town information management, urban integrated pipe network management, battlefield environment simulation and other systems.

\section{KEY TECHNICAL ANALYSIS}

The LOD technology is the abbreviation of Levels of Detail, which means multiple levels of detail. LOD technology refers to the position and importance of the node in the display environment according to the object model. Under the condition that the visual effect of the picture is not affected, the resource allocation of the object rendering is determined by simplifying the detail step by step, and the geometric complexity of the scene is reduced. Thereby improving the efficiency of the rendering algorithm to obtain efficient rendering operations. Shortest path analysis is the most basic and most critical technology for network analysis. It is a research hotspot in computer science, operations research, traffic engineering and geographic information science. The combination of LOD and shortest path technology is applied to the traffic 3D geographic information system, which can effectively solve the fast calculation of the path and the real-time display of the map. The LOD technology and the shortest path analysis model involved in this paper are briefly introduced below. 


\subsection{Terrain LOD Technology}

2.1.1 LOD Technology: LOD (Level of Detail) technology is a multi-scale representation of the scene to achieve a balance between data reduction and loss of realism It selects the appropriate scale model representation to draw based on the distance between the scene object model and the viewpoint. When expressing the model details, if the model is far away from the viewpoint and the projection area of the screen space covers fewer pixels, the model with small scale (rough) is used for representation; on the contrary, the model is closer to the viewpoint and is projected in the screen space. If more pixels are needed to display the details of the model, a large (fine) model of the scale is used for expression.

Based on the map knowledge, setting the model under different scales is an approximation of the real object. The bigger the scale, the closer it is to the real object. Using the function $S$ to represent the approximation, then

$$
S=f(o b j, d)
$$

Where $o b j$ is the real object

$d$ is the distance from the object to the viewpoint

That is, $S$ is a function about $o b j$ and $d . S$ is a continuous decreasing function of the distance $d$. Because $S$ is just an approximation of a real object, there is an error $\delta$ with the real object. When expressed in a mathematical model, there are:

$$
\delta=\int_{0}^{D}[f(x)-S(x, y)] d y
$$

Where $f(x)$ represents the real object

$y$ represents the distance between the viewpoint and the object, and the field of view

The range of variation is $0 \sim D$. The smaller the $\delta$ is, the closer the scale of the model under the expression of the function $S$ is to the real object, and the smaller the gap, and vice versa.

In the actual calculation process, it is necessary to discretize the continuous $S$. The range of view change is divided into $n$ segments, if the distance view is represented as $d_{i}(i=0,1, \ldots n)$. The range $\Delta d_{i}$ of each segment is:

$$
\Delta d_{i}=d_{i}-d_{i-1}(i=1,2, \ldots n)
$$

There is a corresponding $S_{i}$ on $\Delta d_{i}$, and $S_{0}>S_{l}>\bullet \bullet>S_{n}$.

That is to say, the farther the distance is, the smaller the similarity is. Then:

$$
S_{(o b j, d)}=\left\{\begin{array}{cc}
S_{0} & d<d_{0} \\
S_{i-1} & d_{i-1} \leq d<d_{i}, 1<i<n \\
S_{n} & d \geq d n
\end{array}\right.
$$

Then the error function $\delta$ is converted into:

$$
\delta=\sum\left|f(x)-S_{i}\right| \Delta d_{i}
$$

The LOD problem then translates into a problem of $S_{i}$ and $\Delta d_{i}$ that makes $\delta=\min$. Because the $\delta$ function is a generalized general function, it is very difficult to solve it. For better application in engineering, the function needs to be combined with specific requirements and conditions.

\subsubsection{Terrain LOD based on geographic features :} Because LOD is an approximation of true objects at different scales, the application of LOD to terrain-based terrain representation becomes a simplification and synthesis of terrain features. The key issue is the simplification and synthesis of the process of generating multiple resolutions. This simplification process is not intended to remove rough parts from the original model, but to preserve important visual features. The ideal result is a simplified sequence of the initial model. So how to choose a simplified algorithm is the key.

In terms of terrain multi-scale model generation, the current application is mainly based on three methods:

1. Hoppe's 'Progressive Meshes' rule. This method saves the model to the structure - progressive mesh, allowing smooth selection of detail levels based on the current view. The Progressive Meshes algorithm achieves the goal of mesh simplification through a series of edge folding operations, and records the position of the original fixed point and the new vertex and the change of the connection relationship between the fixed points in the simplified process. The point sequence operation can be used to simplify the information sequence according to the reverse tracking, and the deleted model details can be gradually restored, and a simplified model with continuous precision of the original model can be implemented. In fact, this means that the entire model with the lowest level of detail can be displayed at a time, and then more details are gradually displayed.

2. Lindstrom's quad tree approach. The quadtree algorithm starts from the whole complete terrain and recursively divides the terrain into four equal regions. The greater the depth of the segmentation, the higher the resolution obtained. Each of the divided regions is a node, which stores information such as latitude and longitude, height, and the like of a certain region. This method utilizes quadtrees to organize terrain data of different resolutions and utilizes multi-threading for data processing. It uses a quadtree structure to organize terrain data. The no-layer nodes represent terrain blocks of different resolutions. The resolution of each layer node is half of the resolution of its lower nodes, and the leaf nodes are the highest resolution terrain blocks. However, during the process of roaming the terrain block, the program determines which resolution of the terrain block to transfer based on the parameters of the viewpoint, and then performs simplification or optimization processing.

3. Duchaineau's ROAM (Real-time Optimally Adapting Me-shes) rule. The ROAM algorithm is based on a binary tree structure. Each block is a simple equilateral triangle. It is decomposed from the vertex of the triangle to the midpoint of its hypotenuse, and two new equilateral triangles can be generated. Recursively in this way until the desired level of detail is reached.

\subsection{Shortest path analysis}

In geospatial, many natural, artificial linear features form a network with each other. In the shortest path selection, the distance between two points can be defined as the actual 
distance, or it can be positioned as time, freight, traffic, etc. between two points. In other words, it can be defined as the cost of using this edge. Therefore, the shortest path analysis can be performed on different topics. The shortest path problem has a wide range of applications in computers, such as the choice of the shortest path in network communication, and the research of search algorithms in artificial intelligence.

2.2.1 Four commonly used shortest path algorithms: The shortest path algorithm mainly includes Depth First Search, Breadth First Search, Bidirectional Breadth Search and A* algorithm.

The depth first search always extends the deepest node in the current edge of the search tree. After the expansion, they are removed in the edge, and then the search algorithm goes up to the next shallow node that has not yet expanded the successor node. It is characterized by facilitating all branches and finding the optimal solution.

The breadth-first search is a simple search strategy that first extends the root node, then extends all subsequent successors of the root node, then extends their successors, and so on. All nodes of this layer on the search tree have been expanded before any node expansion of the next layer. The Dijkstra algorithm is a typical breadth-first algorithm.

The two-way breadth search is that the search is performed simultaneously in the forward and reverse directions, that is, from the initial node to the target node, and also from the target node to the initial node, when the search in the two directions generates the same child node, terminate the search process.

The $A^{*}$ algorithm uses a heuristic function to evaluate the branches generated during the search process to select the best branch to search.

2.2.2 The $\mathrm{A}^{*}$ algorithm: When studying the shortest path algorithm involved in this paper, the $\mathrm{A}^{*}$ algorithm is used.

The A* algorithm is by far the fastest algorithm for calculating the shortest path, but it is a 'better' algorithm, i.e. it can only find a better solution than an optimal solution, but because of its High efficiency makes it extremely widely used in real-time systems, artificial intelligence, etc.. The $\mathrm{A}^{*}$ algorithm combines heuristics and formal methods. It uses a Heuristic Function to estimate the distance from the current point to the end point (with weights) in the graph, and thus determines its search direction. When this path fails, try other paths. The $\mathrm{A}^{*}$ algorithm formula is expressed as:

$$
f(n)=g(n)+h(n)
$$

Where $\quad f(n)$ is the valuation function of node $\mathrm{n}$ from the initial point to the target point.

$g(n)$ is the actual cost from the initial node to the $\mathrm{n}$ node in the state space. the target node .

$h(n)$ is the estimated cost of the best path from $n$ to

Where $h(n)$ dominates the representation of the $\mathrm{A}^{*}$ algorithm. There are five scenarios:

1. $h(n)=0$ : The $\mathrm{A}^{*}$ algorithm is equivalent to the Dijkstra algorithm and guarantees that the optimal path can be found.
2. $h(n)<$ the distance from the current node to the end point: The $\mathrm{A}^{*}$ algorithm guarantees to find the optimal path. The smaller $\mathrm{h}(\mathrm{n})$ is, the deeper the search depth is.

3. $h(n)=$ the distance from the current node to the end point: The $\mathrm{A}^{*}$ algorithm will only find the best path and can find the result quickly.

4. $h(n)>$ The current distance from the node to the end point: It is not guaranteed to find the optimal path, but the calculation is faster.

5. $h(n)$ is highly correlated with $\mathrm{g}(\mathrm{n})$ : The $\mathrm{A}^{*}$ algorithm becomes BFS (Best First Search) at this time.

This module takes the straight line distance between two nodes as the estimated value, that is,

$$
f=g(n)+\sqrt{(d x-n x)^{2}+(d y-n y)^{2}}
$$

If the value of $g$ is constant, it will be more or less subject to the evaluation value $h$. The node is close to the target point, the $h$ value is small, and the $f$ value is relatively small, which can ensure that the shortest search is performed in the direction of the end point.

\section{APPLIED PRACTICE IN THE FIELD OF TRANSPORTATION}

In practical application, after developing the platform, we realized 3D geographic information and road network generation and real-time display based on LOD technology. The shortest path algorithm is used to calculate the optimal path during vehicle driving, and the navigation of the vehicle is realized. The collision analysis model is used to realize the realtime road condition detection of the simulated driving. The simulation driving is realized by the combination of the driving state and the optimal path of the vehicle; and the simulation driving route is realized and produced in the three-dimensional traffic information system.

\subsection{D road network generation}

Based on the regular grid (GRID) terrain model construction, combined with the shortest path algorithm and manual intervention, the three-dimensional model and display of roads, intersections and surrounding buildings and ancillary facilities in the three-dimensional road network are set up (Fig. 1).

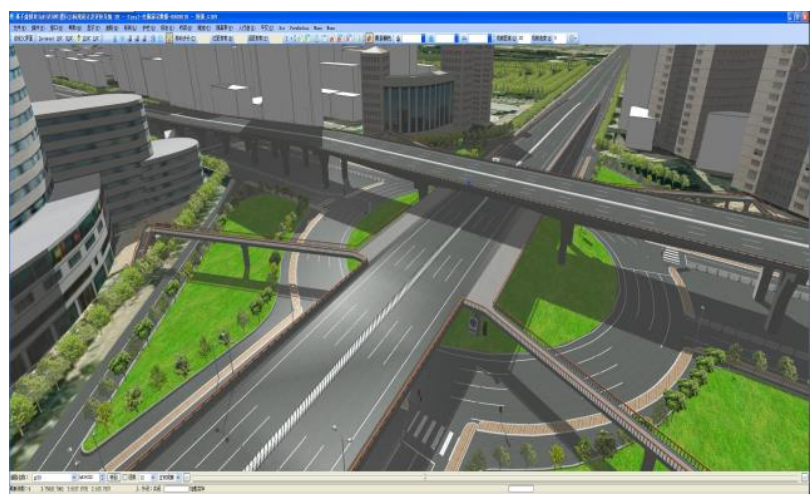

Figure $13 \mathrm{D}$ road network generation effect diagram based on GRID technology 
When implementing a scene by constructing a threedimensional scene through a grid, it is necessary to construct a large number of grids, and when constructing a large-scale scene, it requires a large amount of computer resources. In practical applications, in most cases, it is not necessary to display the entire three-dimensional scene, but according to the needs of roaming, only part of the scene can be displayed to meet the user's needs. Therefore, the technique of batch calling the scene is used to divide the whole scene into different small scene blocks, and the scene blocks are read and called according to the motion change of the roamer viewpoint, so that the viewpoint of the roamer is always located at the center of the reading scene. In the specific implementation, the scene block where the roamer's visual center is located and the neighbouring scene block are transferred into the memory, and then the area to be displayed is determined according to the line of sight and the viewpoint, and displayed in the user's visual scene.

To achieve the above functions, the scene data is first organized according to the block, and the regular grid is used to organize, store and manage the entire scene according to different hierarchical structures. In this way, hierarchical management in data calls is solved, and data access and call volume are reduced. The data is organized in a node tree, and the block data is simplified from top to bottom. In the implementation, a quadtree structure is used to organize the data.
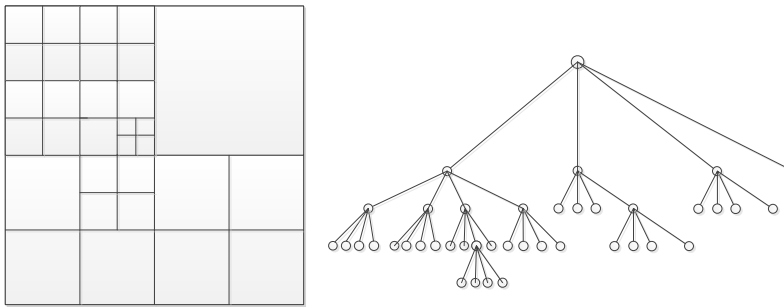

Figure 2 Terrain represented by a quadtree structure

As shown in Figure 2, specify the number of nodes in the terrain model as $\left(2^{n}+1\right)^{2}$.And divided into different levels, so that each node in the tree corresponds to a patch composed of four grid cells. Adjacent sub-scenes are located on adjacent nodes. On the leaf nodes of the node tree, including the texture information, colour information, position information, and the like of the final small area. In this way, each node represents a piece of terrain with different levels of nodes, corresponding terrain areas. The leaf nodes is the highest level of all nodes. Secondly, edge deletion, edge insertion and Boolean matrix are used to solve the crack problem caused by the change of adjacent blocks caused by the quad tree structure.

In the process of calling, the terrain and various information are called together by considering the roughness of the line of sight and the terrain as an indicator, and displayed as needed. Through the above various algorithms, the generation of the three-dimensional road network is realized. The realization of the $3 \mathrm{D}$ road network generation function provides technical support and guarantee for vehicle navigation visualization.

With the support of the database, combined with data precision hierarchical management and spatial indexing technology, the dynamic organization of 3D scene data and the dynamic simplification of $3 \mathrm{D}$ terrain data, so as to have the processing capability of large area large amount of spatial information. We pay particular attention to the support of open data formats in the system development process. The system can support large pyramid data (32 layers), merge terrain data with different precision (Fig. 3), and can automatically smooth and dynamically smooth different terrain data, breaking through the bottleneck of spatial information processing of large data volume.

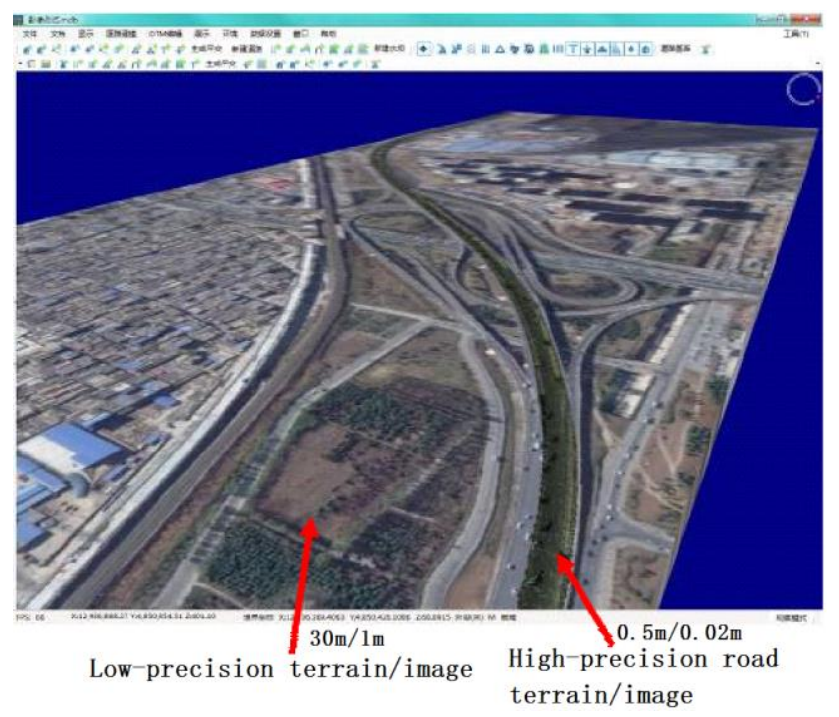

Figure 3 Schematic diagram of fusion and 3D application of high and low precision data

\subsection{Vehicle navigation}

The vehicle navigation function is one of the main functions of the traffic geographic information system. Navigation is divided into static navigation and dynamic real-time navigation. Static navigation is calculated by inputting the origin and destination, and the system automatically calculates the path information in combination with the $\mathrm{A}^{*}$ algorithm. In the system implementation process, according to the improved $A^{*}$ algorithm, according to the valuation function of the temporary marker node:

$$
f *_{(j)}=g_{(j)}+h_{(j)}^{*}+h *_{(j-1)}
$$

Where $g_{(j)}$ is the actual distance from the starting point to the marked node. the end point

$h{ }_{(j)}$ is the minimum distance from the marked node to

$h_{(j-1)}^{*}$ is the minimum distance from the point before the marker node to the target node.

Through this algorithm, the number of traversal of nodes is reduced, the search speed is improved, and the path calculation is completed. Then inform the vehicle about the events that should be noticed in front of the trip and the best route and distance to the destination. Through the above algorithms and processes, the static navigation function of the vehicle is realized.

Dynamic real-time navigation is to use GNSS to obtain the position and three-dimensional posture and driving direction of the vehicle in real time or at regular intervals, and to go down according to the actual conditions of the road, such as road construction, blockage, and wrong way (when the vehicle deviates from the original calculation path by 200 meters or 
more). Construction, blockage or original calculation of the route and calculate the new shortest path, refresh correction on the display to ensure that the navigation function is continuous, fast and convenient. At the same time, with the voice prompts, the driver can use both ears and eyes to drive easily.

The vehicle navigation realizes the accurate calculation and simulation of the position and route of the vehicle on the road, and realizes the system guarantee for the simulated driving.

\subsection{Simulated driving}

3.3.1 Main content of simulated driving: Based on LOD technology to realize the management and organization of virtual three-dimensional spatial data, it is convenient to realize the simulation of the actual traffic road network, realize the visual special effects in the GIS system, complete the virtual reality road conditions, and lay the simulation data foundation for the simulation driving. On this basis, electronic control technology is used to control the variable acceleration process of the vehicle (including the size and direction of the speed) to realize human-like driving. There are many factors to consider during the exercise of the vehicle, the most important of which is the vehicle dynamics model (including vehicle speed model, direction control model, braking force model, resistance model ), in addition to the above models. Some other related technologies, such as vehicle dispatching systems, communication systems, and human-computer interaction systems, are also needed.

3.3.2 Vehicle collision model : The comprehensive application of LOD technology, dynamic model and automobile collision model is adopted. In the design process, the vehicle is controlled by keyboard, steering wheel, handle, etc., and the linear vehicle model is constructed by adjusting the angle of view and tracking the scene to realize the simulated driving of the vehicle. The system realizes the functions of rear-view mirror, eagle eye view and so on during the simulation. Collision detection for simulated driving is implemented according to the bounding box method. This method uses a bounding box with simple geometric features but a slightly larger volume to describe complex geometric objects. By constructing a tree-like hierarchy to approximate the model, in the process of traversing the model tree, quickly test the intersection of the bounding boxes and eliminate disjoint as early as possible. The element only performs further intersection test on the overlapping part of the bounding box, completes the collision detection, and calculates the relevant dynamic parameters required for driving.

3.3.3 Vehicle direction control model and braking force model : In the actual operation, the simulated driving is mainly in the aspects of adding (subtracting) speed, parallelizing, turning, etc. Firstly, the LOD technology is used to realize the information loading and display of the road where the vehicle is located, and then the road condition is detected according to the vehicle collision model of the driving vehicle. The relevant operation instructions are issued for simulation, and the driverless driving of the vehicle can be realized to some extent.

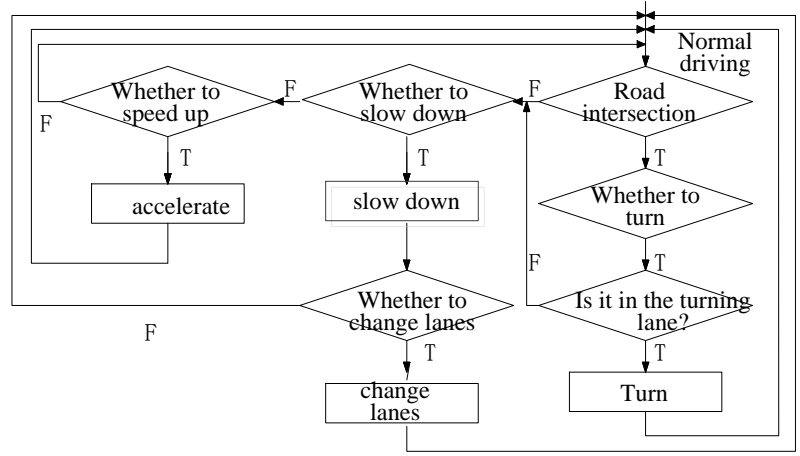

Figure 4. Simulation driving implementation flow chart

1. Adding (minus) speed, first adjust the angle of view, use LOD technology to expand the road information display range, and then use the collision detection technology to judge the intersection test between the vehicle and the surrounding vehicles, roads and road auxiliary facilities, and the distance parameters obtained according to the collision detection. Calculate the acceleration (decrease) speed, call the speed model, achieve fuelling, increase speed (or brake, downshift, deceleration), and complete the operation.

2. When the vehicle is connected, the LOD technology is used to realize the loading and display of the detailed information of the left and right lanes of the vehicle. The collision detection technology is used to detect the intersection detection of the lane and the lane, and the safe distance of the lane is calculated. Then, calculate the vehicle according to the kinetic model and judge whether the vehicle can be connected and the line speed. If the operation can be carried out within the safety threshold, if the line is not suitable, wait and re-detect the determination until the line operation is completed.

3. Turning the vehicle, according to the navigation module system prompt, if it is necessary to turn, the system first uses the LOD technology to transfer relevant road information to complete the collision detection. Determine whether the vehicle is in the turning lane, calculate the turning radius and turning speed of the vehicle, call the speed model and the direction control model, use the parameters obtained by the collision detection to decelerate, control the steering light to indicate the steering, and implement the steering operation.

Combined with the simulation driving function, LOD technology and shortest path technology have realized related functions and applications in the "Virtual Reality Based Road Traffic Marking Design and Evaluation System" of the Ministry of Communications Highway Science Research Institute.

\section{CONCLUSION}

The application of LOD technology and shortest path technology in traffic 3D geographic information system provides technical support for solving 3D modelling, display and visualization. GIS based on this technology can clearly and intuitively display the road and its ancillary facilities and surrounding environment. Combining navigation technology and simulated driving technology to realize intelligent vehicle traffic information system can solve road traffic safety problems caused by driver human factors; by simulating real road conditions, it can effectively shorten driving distance, increase road capacity and improve road operation efficiency; It is 
possible to replace the driver in special circumstances to ensure safe passage of the vehicle. Through the development of the actual 3D GIS platform for the LOD technology and the shortest path algorithm, this paper realizes the road network three-dimensional display, simulated navigation and simulated driving on the real situation of the road during the driving process of the vehicle, and takes the LOD technology and the shortest path algorithm in the traffic. This is the first step in the application of 3D GIS. With the continuous improvement and application of LOD technology and shortest path algorithm, this technology will be able to provide a broader technical guarantee for intelligent transportation, providing more security and convenience for our lives.

\section{REFERENCES}

Andrew S. Tanenbaum, 1998. Computer Networks (Third Edition), Prentice Hall International, Inc., 281-285.

Bai, Q.X., 2007b. Design and Implementation of Vehicle Navigation System Based on eSuperMap, Xi'an: Northwest University, Dissertation, 26-27.

Cai, Z.F., Zhang, A.Y., 2002a. Research on Vehicle Simulation Driving Model and Simulation, Journal of Zhejiang University (Engineering Edition), 36(3), 327-330.

CHEN, J., JIANG, H.F., 2009a. Design of 3D Simulation Evaluation System for Road Traffic Signs Based on Virtual Reality Technology. Journal of Highway and Transportation Technology, 5(03),40-43.

Chen, L., 2010b. Design and Implementation of 3D Simulation Car Navigation System. Chengdu: University of Electronic Science and Technology, Dissertation.47-49.

Chen, Y.K., 2004b. Visualization of large data volume scenes in 3DGIS. China Graduate School of Science (Research Institute of Remote Sensing),11-19.

Clark J., 1976a. Hierarchical geometric models for visible surface algorithms. Communications of the ACM, 19(10), 547554 .

Duan, L.Q., Zhu, J.J., Wang, Q.S., Ma, L., 2004a. Improved Shortest Path Search A* Algorithm Efficient implementation. Ocean Mapping, 05, 20-22.

Lei, J.H., Zeng, F.X., Wu, M.X., 2010a. Research on viewpoint-related LOD terrain simulation algorithm based on quadtree. Manufacturing Automation, 08,211-214+228.

Li, A.D., Yin, N.D.,2008a. Automobile Driving Simulator Research on Motion Model. Journal of Huangshi Institute of Technology,24(2),26-30 .

Li, Z.L., Zhu, Q., 2001. Digital Elevation Model. Wuhan: Wuhan University Press, 7,3-7.

Liu, K., Zhou, Y., 2013a. Research on the Application Prospect of 3D Geographic Information System in Smart Cities. Urban Architecture, (14),295.

Lu, K.C., Lu, H.M., 1982. Graph Theory and Its Applications (Second Edition), Beijing: Tsinghua University Press, 85-90.

Shen, Y.B., 2005a. Research and Application of Collision Detection Technology in Virtual Environment. Journal of Transportation and Computer, 23(1), 74-78.
Shu, L.C., 2011a. 3D GIS-based mine 3D visualization platform. Industrial and Mining Automation, 37(06),7-11.

Tan, B., 2003. Research on large-area terrain visualization technology . Journal of Image and Graphics, 8A(5), 578-585.

Wang, C.X., Huang, T., 2013a. Application of Shortest Path Algorithm in Campus Geographic Information System. Journal of Changchun Teachers College, 32(12),39-40+19. 